\title{
A comparison of biomarker responses in juvenile diploid and triploid African catfish, Clarias gariepinus, exposed to the pesticide butachlor
}

\begin{abstract}
Influence of waterborne butachlor (BUC), a commonly used pesticide, on morphometric, biochemical, and molecular biomarkers was evaluated in juvenile, full sibling, diploid and triploid African catfish (Clarias gariepinus). Fish were exposed for 21 days to one of three concentrations of BUC [mean measured $\mu \mathrm{g} / \mathrm{L}: 22,44$ or 60 ]. Unexposed (control) triploids were heavier and longer and had higher visceral-somatic index (VSI) than diploids. Also, they had lighter liver weight (HSI) and showed lower transcript levels of brain gonadotropinreleasing hormone $(\mathrm{GnRH})$, aromatase (cyp191b) and fushi tarazu-factor (ftz-f1), and plasma testosterone levels than diploids. Butachlor treatments had no effects, in either diploid or triploid fish, on VSI, HSI, weight or length changes, condition factor (CF), levels of plasma testosterone, 17- $\beta$ estradiol (E2), cortisol, cholesterol, or mRNA levels of brain tryptophan hydroxylase (tph2), forkhead box L2 (foxl2), and $11 \beta$-hydroxysteroid dehydrogenase type 2 (11 -hsd2). Expressions of cyp191b and ftz-f1 in triploids were upregulated by the two highest concentrations of BUC. In diploid fish, however, exposures to all BUC concentrations decreased GnRH transcription and the medium BUC concentration decreased $\mathrm{ftz}-\mathrm{f1}$ transcription. Substantial differences between ploidies in basal biomarker responses are consistent with the reported impaired reproductive axis in triploid C. gariepinus. Furthermore, the present study showed the low impact of short term exposure to BUC on reproductive axis in C. gariepinus.
\end{abstract}

Keyword: Biochemical biomarkers; Gene; Morphometric parameters; Polyploid; Steroid 